\title{
EDITORIAL
}

For reprint orders, please contact: reprints@futuremedicine.com

\section{Can being bilingual affect the onset of dementia?}

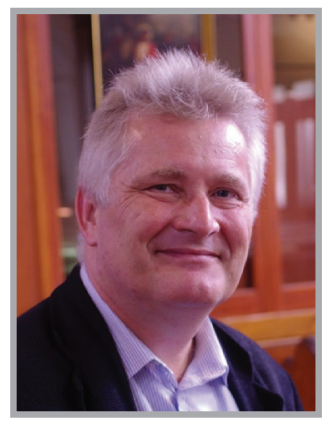

Thomas $\mathrm{H} \mathrm{Bak}{ }^{* 1,2}$

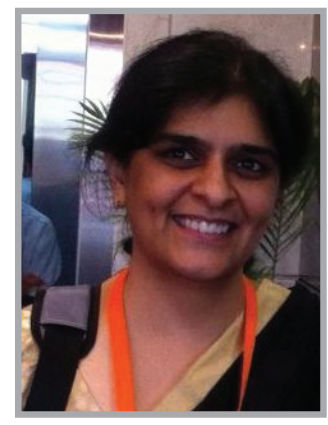

Suvarna Alladi ${ }^{3}$
"Converging evidence from

studies in children, healthy

elderly and demented

patients points in the same

direction: bilingualism has a positive effect on cognition throughout the lifespan."
The dramatic increase in the proportion of elderly people in the world population has led to a growing importance of age-related diseases, in particular dementia. Importantly, this development is not confined to the western world. Dementia is recognized as a major health issue in India, China and many other countries across all continents. It has been described as a "public health priority" by the WHO [1] and became the topic of the last meeting of the G8 economic superpowers in London (UK) in December 2013.

Given the rather limited success of the currently available drug therapies for dementia, much interest has been directed towards potential risk factors, but also towards potential protective factors against it. An influential concept in this respect is that of cognitive reserve, assuming that a better level of premorbid functioning may delay the onset or alleviate the manifestations of cognitive decline. Many studies have documented an association between physical, mental and social activity and better cognitive function in old age [2]. From scientific journals to popular press, broad claims have been made regarding 'brain boosting' effects of crossword puzzles, training programs or video games. However, one type of mental activity seems to generate more controversy than others: bilingualism.

The research on the relationship between bilingualism and dementia started explicitly with the seminal observation by Bialystok et al. that bilinguals develop dementia 4 years later than monolinguals [3]; a finding later confirmed by another study from the same Toronto-based research group [4]. However, these findings do not come out of the blue, but are a logical extension of the emerging field of bilingualism and cognition research. Cognitive advantages of bilingualism have been detected in infants as young as 7 months [5] and have been extensively documented in children [6]. At the other end of the life spectrum, the number of languages spoken has been associated with cognitive function in a large study of healthy elderly [7]. The results of another Canadian study from Montreal paint a slightly more complex picture, with a bilingualism effect detectable in the immigrant group

\section{KEYWORDS}

- bilingualism • cognitive aging

- cognitive reserve $\bullet$ dementia

- monolingualism

'School of Philosophy, Psychology \& Language Sciences (PPLS) \& Centre for Cognitive Aging \& Cognitive Epidemiology (CCACE), University of Edinburgh, 7 George Square, Edinburgh, EH8 9JZ, UK

${ }^{2}$ Centre for Clinical Brain Sciences (CCBS), 49 Little France Crescent, Edinburgh, EH16 4SB, UK

${ }^{3}$ Department of Neurology, Nizam's Institute of Medical Sciences, Hyderabad, India

*Author for correspondence: thomas.bak@ed.ac.uk

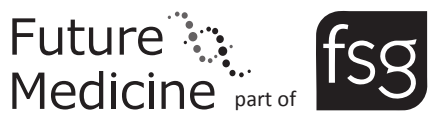




"Bilinguals negotiate
constantly between
different sounds, words,
concepts, grammatical
structures and social
norms associated with
the languages they
speak. This is bound to
provide permanent,
intensive and versatile
mental training."

"Bilinguals negotiate constantly between different sounds, words, concepts, grammatical structures and social the languages they peak. This is bound to mental training."
mentive and versatile and a multi- but not bi-lingualism effect in the autochthonous population [8].

The results of Chertkow et al. point to a fundamental problem of the early studies of bilingualism and dementia: the difficulty of separating the effects of bilingualism and immigration [8]. If most bilingual patients are first- or second-generation immigrants, but most monolingual ones are derived from the autochthonous population, a difference between both groups may be owing not only to language, but also to other factors, such as different ethnic background, education, lifestyle, diet, attitude to health and health services, among others. Indeed, immigration itself could be viewed as a confounding factor, since those who have successfully migrated and settled in a new country are not necessarily a representative sample of their source population. However, these limitations have been addressed by a recent study from Hyderabad (India) [9]. In Hyderabad, as in many other regions of India, bilingualism has been part of everyday life for centuries. Accordingly, both mono- and bi-lingual groups were recruited from the same autochthonous population, eliminating the confounding variable of immigration. The results were stunningly similar to that from Toronto: 4-5 years later onset of dementia in the bilingual group when compared with the monolingual one. To address another potential confounding variable, that of differences in education [10], a separate analysis was conducted in illiterate patients: here the bilingual advantage was even larger, reaching 6 years. Finally, a bilingual advantage was found separately in three different types of dementia: frontotemporal dementia, Alzheimer's disease and in vascular dementia.

Obviously, further studies are necessary to confirm the influence of bilingualism on dementia and to explore the nature of this relationship. However, the converging evidence from studies in children, healthy elderly and demented patients points in the same direction: bilingualism has a positive effect on cognition throughout the lifespan. So why is this idea so difficult to accept? Why should knowing and using several languages in everyday life have less influence on cognitive function than playing a computer game? In our view the roots of this skepticism are not so much due to insufficient evidence in favor of bilingualism, but rather to many misconceptions associated with it.

The first and most fundamental one is to view bilingualism as a rare and unusual phenomenon, maybe in need of scientific study by selected specialists, but of little relevance to the wide population. Such a view typically results from an exclusively western perspective. In many western and, in particular, English-speaking countries, bilingualism tends to be associated with one of three options: membership of linguistic minorities, immigration or acquisition of foreign languages at school. What all three options have in common is that they tend to be perceived as unusual, different from the norm. By contrast, monolingualism is defined as default, the 'normal state' of human mind and brain. Scientifically, there is little to support such an attitude. Bilingualism is very common worldwide and there are reasons to believe that it may have been the original state of human communities [11]. Indeed, the ease with which children can acquire multiple languages [12] suggests that our brains are extremely well suited to support it. In large parts of the world, such as in Hyderabad, it is bilingualism that can be defined as default and monolingualism as an unusual phenomenon. People growing up and living in such places are exposed to multiple languages throughout their lives, from childhood to old age. Being bilingual is for them the natural state. Accordingly, the title of our recent paper could be more controversially reformulated into 'Monolingualism accelerates the onset of dementia'. Given the fact that the majority of our patients (as well as the majority of the population they were coming from) were bilingual, this would have been arguably a more appropriate statement.

The second aspect of bilingualism, which may be important for its effect on cognition, is its holistic nature. It cannot be reduced to one task or one cognitive function. Bilinguals negotiate constantly between different sounds, words, concepts, grammatical structures and social norms associated with the languages they speak. This is bound to provide permanent, intensive and versatile mental training. Current research in this field focuses on selective attention, attentional switching, response inhibition and other executive functions [13-17]. However, as important as this research is to elucidate the effects of bilingualism on normal and abnormal cognitive functions, it might not capture all of it. Like in other areas of medicine, or indeed science in general, we need to be prepared to venture beyond the consensus established in systematic reviews and search for the unexpected [18]. Future studies are likely to unearth many other ways in which bilingualism affects mental processes. 
The third and final aspect of bilingualism we would like to discuss here is its diversity [19]. As discussed above, bilingualism can, but does not have to be associated with national, ethnic, social or religious minorities, with immigration and displacement, with particular education or with intermarriage between people speaking different languages. Bilinguals may have a higher, but also a lower social status than their monolingual counterparts. Different languages can be acquired in the family, at school, at work or in everyday life on the street or in the village. They can be learned simultaneously or consecutively. They can be used with the same bilingual people (with a high degree of switching) or in strictly separate social contexts. The level of proficiency can vary considerably, from basic ability to communicate to a perfect, native-like command. All these factors are likely to influence the way in which bilingualism shapes cognitive processes and indeed their underlying neural substrates [20].

Bilingualism cannot be treated, therefore, as a unitary phenomenon. What we need are comparative studies of different types of bilingualism. Such a diversification of research is bound to produce at some stage conflicting results.

\section{References}

1 WHO. Alzheimer's Disease International (2012) Dementia: a Public Health Priority. WHO, Geneva, Switzerland (2012).

2 Plassman BL, Williams JW, Burke JR, Holsinger T, Benjamin S. Systematic review: factors associated with risk for and possible prevention of cognitive decline in later life. Ann. Intern. Med. 153(3), 182-193 (2010).

3 Bialystok E, Craik FI, Freedman M. Bilingualism as a protection against the onset of symptoms of dementia. Neuropsychologia 45(2), 459-464 (2007).

4 Craik FI, Bialystok E, Freedman M. Delaying the onset of Alzheimer disease Bilingualism as a form of cognitive reserve. Neurology 75(19), 1726-1729 (2010).

5 Kovács ÁM, Mehler J. Cognitive gains in 7-month-old bilingual infants. Proc. Natl Acad. Sci. USA 106(16), 6556-6560 (2009).

6 Barac R, Bialystok E. Cognitive development of bilingual children. Language Teaching 44(1), 36-54 (2011).

7 Kavé G, Eyal N, Shorek A, Cohen-Mansfield J. Multilingualism and cognitive state in the oldest old. Psychol. Aging 23(1), 70 (2008).

Bilingualism should not be misunderstood as a 'cognitive panacea'. It is only one of many factors influencing cognitive functions and dementia and its influence can be either strengthened or weakened by other variables, be it genetic or environmental. Future studies of cognitive impact of bilingualism will need to characterize the type of bilingualism, the age, the manner of acquisition and the patterns of use, among others. However, with larger and better studies from a larger number of countries and cultures a clearer picture will finally emerge, allowing us to better understand the interaction between bilingualism, cognitive functions and dementia. Given the huge potential benefits at extremely low costs it is certainly a topic worth pursuing.

\section{Financial \& competing interests disclosure}

The authors have no relevant affiliations or financial involvement with any organization or entity with a financial interest in or financial conflict with the subject matter or materials discussed in the manuscript. This includes employment, consultancies, honoraria, stock ownership or options, expert testimony, grants or patents received or pending, or royalties.

No writing assistance was utilized in the production of this manuscript.

8 Chertkow H, Whitehead V, Phillips N, Wolfson C, Atherton J, Bergman H. Multilingualism (but not always bilingualism) delays the onset of Alzheimer disease: evidence from a bilingual community. Alzheimer Dis. Assoc. Disord. 24(2), 118-125 (2010).

9 Alladi S, Bak TH, Duggirala V et al. Bilingualism delays age at onset of dementia, independent of education and immigration status. Neurology 81(22), 1938-1844 (2013).

10 Gollan TH, Salmon DP, Montoya RI, Galasko DR. Degree of bilingualism predicts age of diagnosis of Alzheimer's disease in low-education but not in highly educated Hispanics. Neuropsychologia 49(14), 3826-3830 (2011).

11 Evans N. Dying Words: Endangered Languages and What They Have to Tell Us. Wiley-Blackwell, West Sussex, UK (2009).

12 Bialystok E. Language Processing in Bilingual Children. Cambridge University Press, Cambridge, UK (1991).

13 Bialystok E, Viswanathan M. Components of executive control with advantages for bilingual children in two cultures. Cognition 112(3), 494-500 (2009).
14 Hilchey MD, Klein RM. Are there bilingual advantages on nonlinguistic interference tasks? Implications for the plasticity of executive control processes. Psychon. Bull. Rev. 18(4), 625-658 (2011).

15 Bialystok E, Craik FI, Luk G. Bilingualism: consequences for mind and brain. Trends Cogn. Sci. 16(4), 240-250 (2012).

16 Costa A, Hernández M, Costa-Faidella J, Sebastián-Gallés N. On the bilingual advantage in conflict processing: now you see it, now you don't. Cognition 113(2), 135-149 (2009).

17 Green DW. Language control in different contexts: the behavioral ecology of bilingual speakers. Front. Psychol. 2, 103 (2011).

18 Bak TH. The importance of looking in dark places. Amyotroph. Lateral Scler. Frontotemporal Degener. 14(1), 1-2 (2013).

19 Luk G, Bialystok E. Bilingualism is not a categorical variable: interaction between language proficiency and usage. J. Cogn. Psychol. 25(5), 605-621 (2013).

20 Klein D, Mok K, Chen J-K, Watkins KE. Age of language learning shapes brain structure: a cortical thickness study of bilingual and monolingual individuals. Brain Lang. doi:10.1016/j.bandl.2013.05.014 (2013) (Epub ahead of print). 